\title{
Troponina T vs Troponina I como Valor Predictivo para Síndrome Coronario Agudo en menores de 80 Años
}

\section{Troponin T vs Troponin I as Predictive Value for Acute Coronary Syndrome in Children Under 80}

\author{
Pla Cala PE ${ }^{1}$, Polo Barranco EA루, Peñaloza Parra S2, Cadena Bonfanti A. ${ }^{3}$ \\ ${ }^{1}$ Universidad Libre (Col), ${ }^{2}$ Secretaria de Salud Departamental Atl. (Col), ${ }^{3}$ Clínica de la Costa (Col),
}

Resumen - Actualmente en la injuria miocárdica, ha tenido gran relevancia clínica la utilidad de las troponinas como método diagnóstico, repercutiendo en el manejo del síndrome coronario agudo.

Objetivo: Analizar la importancia de los biomarcadores troponina Te I y otros elementos adyuvantes que cumplen un papel trascendental como es el electrocardiograma y los diferentes métodos invasivos, el cual brinda una orientación para la concepción de un diagnóstico oportuno que permita tomar las acciones pertinentes en un paciente con síndrome coronario agudo.

Materiales y M étodos: Estudio descriptivo comparativo en 35 pacientes, adultos mayores de 21 años y menores 80 años, que se encontraban hospitalizados en la Empresa Social del Estado Centro de Atención y Rehabilitación Integral - E.S.E CARI de Alta complejidad, en el periodo comprendido entre el año 2012 a 2014.

Resultados: Los pacientes presentaron síndrome coronario agudo (infarto agudo de miocardio con o sin elevación del ST), con factores de riesgo cardiovascular tales como: sedentarismo, tabaquismo, hipertensión arterial, diabetes, valvulopatías, dislipidemia y antecedentes de cardiopatía isquémica. El punto a analizar fue la elevación de los biomarcadores troponina T y troponina I en un periodo menor y mayor a 3 horas, desde el inicio de los síntomas (dolor torácico, criodiaforesis, disnea) hasta el ingreso a la institución hospitalaria. Se compararon los resultados obtenidos de cada uno de estos biomarcadores en el diagnóstico precoz y oportuno del síndrome coronario agudo. De las muestra analizada ( $n=35$ pacientes) se obtuvo que la troponina $\mathrm{T}<3$ horas se elevó un $76.4 \%$ en rangos de edad entre los 60-80 años en contraste con troponina I $<3$ horas en ese mismo grupo etario que fue de $70.5 \%$.

Conclusiones: se evidenció que ambos biomarcadores son equiparables con una ligera ventaja de la troponina T con respecto a la I.

Palabras Clave: Síndrome coronario agudo, troponina, biomarcadores, anginainestable, infarto agudo de miocardio.

Abstract - Currently in the myocardial injury, the usefulness of troponins as a diagnostic method has had great clinical relevance, affecting the management of acute coronary syndrome.

Objective: Analyze the importance of the troponin T and I biomarkers and other adjuvant elements that play a transcendental role such as the electrocardiogram and the different invasive methods, which provides an orientation for the conception of a timely diagnosis that allows taking the pertinent actions in a patient with acute coronary syndrome. Materials and methods: Comparative descriptive study in 35 patients, adults aged 21 years and younger 80 years, who were hospitalized in the Social Enterprise of the State Center for Comprehensive Care and

Correspondencia: Paul Eduardo Pla Cala. Facultad de Salud. Universidad Libre. Km 7 Ant. Vía Pto Col. Barranquilla, Atlántico, Colombia. daribogf@hotmail.com. Citar:

Pla Cala PE, Polo EA, Peñaloza S, Cadena A. Nivel de Cumplimiento de Troponina T vs Troponina I como Valor Predictivo para Síndrome Coronario Agudo en menores de 80 Años. Cienc. innov. salud. 2015; 3 (2):4 - 10.
Rehabilitation - ESE CARI of high complexity between 2012 to 2014. Results: Patients presented with acute coronary syndrome (acute myocardial infarction with or without ST elevation), with cardiovascular risk factors such as: sedentary lifestyle, smoking, hypertension, diabetes, valvulopathies, dyslipidemia and a history of ischemic heart disease. The point to be analyzed was the elevation of the biomarkers T and I troponin in a period shorter and longer than 3 hours, from the onset of symptoms (chest pain, cryodiaphoresis, dyspnea) until admission to the hospital. Results of each of these biomarkers were compared in the early and timely diagnosis of acute coronary syndrome. Of the analyzed samples (n $=35$ patients), troponin $\mathrm{T}<3$ hours was found to increase by $76.4 \%$ in age ranges between $60-80$ years in contrast to troponin I $<3$ hours in the same age group, which was $70.5 \%$.

Conclusions: It was demonstrated that both biomarkers are comparable with a slight advantage of $T$ troponin with respect to I troponin.

Keywords: Acute coronary syndrome, troponin, bio-markers, angina-line, acute myocardial infarction.

\section{INTRODUCCIÓN}

El Síndrome coronario agudo (SCA) se encuentra entre las patologías más frecuentes que presentan los pacientes que acuden a un servicio de urgencias. La evaluación de los pacientes con esta entidad es un reto para los facultativos, dada la variedad de las causas potenciales y afecciones clínicas asociadas, como diabetes mellitus, dislipidemias, obesidad y antecedentes de cardiopatías previas, jugando un papel importante en el desarrollo del síndrome coronario agudo y esto asociado a los estilos de vida no saludable (sedentarismo, tabaquismo, dieta rica en grasa saturada, alta en sodio y baja en potasio).

En estados Unidos y Europa la mortalidad por cardiopatía isquémica se ha mantenido, se estima que en España se producen 68.500 infartos agudos al miocardio (IAM ) por año, de los que so lo 41.000 llegarían al hospital. El IAM representa el $10 \%$ de las altas hospitalarias y el $25 \%$ de los síndromes coronarios en urgencias (2). El $65 \%$ de las muertes por IAM se producen fuera del hospital y 
en las unidades coronarias la mortalidad es del $10 \%$ a los 28 días, aumentando con la edad. La muerte súbita constituye entre el 2 y el $12 \%$ de los fallecimientos en España. El $80 \%$ son de origen cardíaco y se producen entre 19 y 159 casos por 100.000 hombres al año y entre 2 y 35 por cada 100.000 mujeres. Por su parte en los servicios de urgencia hospitalarios de EE.UU. se estima que refieren dolor torácico no traumático el 27,7 de pacientes por 1.000 habitantes año, y que entre el $11 \%$ y el $17 \%$ corresponden a SCA ( 4 casos por 1.000 habitantes año) cifra muy inferior de la estimada en nuestro medio donde la prevalencia de la cardiopatía isquémica, se estima en 8,6 casos por 1.000 habitantes al año $(3,4)$.

El panorama a nivel nacional no es muy alentador es por esto que sociedades científicas como la asociación colombiana de medicina interna y la sociedad colombiana de cardiología han establecido al síndrome coronario como la primera causa de muerte por enfermedades crónicas no transmisibles. El riesgo de desarrollar enfermedad coronaria a lo largo de la vida después de los 40 años es $49 \%$ para hombres y $32 \%$ para mujeres (5). En el estudio ARIC (riesgo de arteriosclerosis en comunidades, por su sigla en inglés) del NHLBI, la incidencia promedio ajustada por edad para enfermedad coronaria por 1000 personas/año es 12,5 , hombres blancos; 10,6 , hombres negros; 4.0 , mujeres blancas, y 5.1 para mujeres negras el promedio de edad del primer IAM es 65.8 años para los hombres y 70.4 años para las mujeres. La incidencia de SCA en mujeres después de la menopausia es 2 a 3 por cada mujer (6).

Las pruebas para medir los marcadores cardíacos deben estar disponibles para el médico las 24 horas del día, y los siete días de la semana; además es muy importante que los resultados puedan entregarse al médico solicitante en un espacio muy corto de tiempo. Algunas de estas pruebas pueden realizarse a la cabecera del enfermo ya sea en los servicios de urgencias o en la unidad de hospitalización donde esté ingresado el paciente (7). A menudo se realizan determinaciones seriadas de uno o varios marcadores cardíacos para estar seguro de que se detectará cualquier pequeño aumento de su concentración, y para estimar la gravedad del infarto de miocardio (7).

Esta investigación tuvo como propósito comparar cuantitativamente los biomarcadores de inflamación como troponinas cardiacas $T$ e I, que permitan evaluar de manera rápida y oportuna los pacientes con SCA, estratificando el riesgo de los pacientes del Servicio de
Urgencias(8).

Además esto abre una ventana para que investigaciones futuras sigan incursionando en la utilidad de los biomarcadores como métodos de laboratorio relevantes en el síndrome coronario agudo y de esta manera mejorar las impresiones diagnósticas (2).

Con la aparición de escalas encaminadas a evaluar el riesgo cardiovascular identificando variables como: edad, sexo, tabaquismo, perfil lipídico, cifras tensionales, comorbilidades y la importancia del colesterol no HDL dieron lugar al nacimiento de estudios como el framinghan y UKPDS. Que han permitido estadificar de manera temprana los riesgos de los pacientes con enfermedades cardiovasculares $(1,2)$.

En el SCA el marcador que ha merecido más atención en la última década son las troponinas. El reconocimiento de la importancia de la inflamación en el desarrollo de la enfermedad arteriosclerótica en general y de los SCA en particular ha sido paralelo al interés por las troponinas. Lo que crea el siguiente interrogante, como formulación de la problemática:

Pese a los nuevos avances tecnológicos que se encuentran disponibles en la actualidad como "electrocardiogramas, ecocardiogramas modo M bidimensional y transesofágicos, estudios de medicina nuclear y los biomarcadores tisulares de injuria miocárdica (troponina T y troponina I), se presentan retrasos en el diagnóstico y posterior tratamiento de los pacientes, traduciéndose en un aumento de la morbimortalidad de esta patología (1)".

Es importante tener a disposición los biomarcadores de inflamación que permita evaluar el diagnóstico temprano y el pronóstico de los pacientes con SCA (2). De esta forma tener a la mano métodos menos costosos e invasivos para el paciente. La troponina T y la troponina I son proteínas que se liberan en el organismo en respuesta a la lesión aguda, infección u otros estímulos inflamatorios presente en las paredes arteriales afectadas en el proceso de formación del ateroma (3); ruptura de placas ateromatosas y desarrollo de eventos clínicos aterotrombóticos $(2,3)$.

Por esta razón se hace necesario desarro llar un estudio de investigación para un diagnóstico precoz y oportuno que al mismo tiempo permita realizar seguimiento de los pacientes utilizando estos dos biomarcadores (2). De esta manera se puede determinar cuál de los dos es más 
relevante y confiable a la hora de diagnosticar y medir morbimortalidad a futuro.

Además hay que tener en cuenta el efecto negativo que tiene el SCA en la vida útil de los pacientes, en el entorno familiar y su reincorporación a la sociedad, que muchas veces está limitada. Presentándose un aumento en las tasas de recidivas de reinfartos e incremento de trastornos psicológicos como depresión, ansiedad y conflictos familiares generadas por las secuelas de esta patología.

Lo que lleva plantear posibles hipótesis como los son: Los biomarcadores troponina $T$ y troponina I son útiles como factor diagnostico o pronostico en pacientes con síndrome coronario agudo (3). La utilización de biomarcadores troponina $\mathrm{T}$ y troponina I no son útiles como factor diagnostico o pronostico en pacientes con síndrome coronario agudo. Y una tercera hipótesis seria determinar si el valor de la troponina $T$ es mejor valor predictivo positivo que la troponina I en el síndrome coronario agudo.

\section{ATERIALESY MÉTODOS}

Se realizó una investigación cuantitativa, sobre 35 pacientes que ingresaron a la E.S.E CARI de Barranquilla en el periodo comprendido entre el 2012-2014 con síndrome coronario agudo, con factores de riesgo de tabaquismo, diabetes, hipertensión arterial, valvulopatías, dislipidemia y antecedentes de cardiopatía isquémica, que cumplían con los criterios de inclusión. Se determinaron a los pacientes los valores de troponina $T y$ troponina I y su correlación con el tipo de síndrome coronario agudo; se evaluó el tiempo de elevación de la troponina T y troponina I en el SCA y la asociación de elevación de estos relacionados a las complicaciones cardiacas post evento coronario, mediante variables determinadas por diversos factores como el tabaquismo, el sedentarismo, diabetes, sexo edad, hipertensión arterial, entre otros. Igualmente se analizaron diferentes factores de riesgo cardiovascular que inciden directamente en la elevación de los biomarcadores troponina T e I en la población de estudio.

Se estableció la correlación entre los valores de troponina T, troponina I y el tipo de Síndrome coronario agudo; estipulando las similitudes y diferencias en algunos casos. Se estimó el tiempo de elevación de la troponina $\mathrm{T}$, troponina I en el síndrome coronario agudo y la asociación de elevación de troponina $T$, troponina I con relación a las complicaciones cardiacas post evento coronario.

Las variables fueron estandarizadas a rangos para comparar los resultados y hacer los cruces correspondientes. Las cuales fueron agrupadas y tabuladas utilizando las medidas de tendencia central. Para establecer la significancia de los cruces estadísticos, se realizó una prueba de $\chi^{2}$, así como un análisis de correspondencia simple para medir la asociación conjunta de las variables y sus niveles. Los análisis estadísticos fueron realizados en R-CRAN.

\section{RESULTADOS}

El $42,86 \%$ de los pacientes con Infarto Agudo al Miocardio no presentaron elevación de ST. Para ambas Troponinas lgual porcentaje de elevación después de las 3hrs como menor a las $3 \mathrm{hrs}$. El valor porcentual para Elevación del ST con elevación de Troponina T menor a $3 \mathrm{hrs}(40,00 \%)$ fue mayor que en la Troponina I (31,43\%). El infarto con mayor frecuencia fue el que presento Elevación del ST para ambas Troponinas (57,14\%).

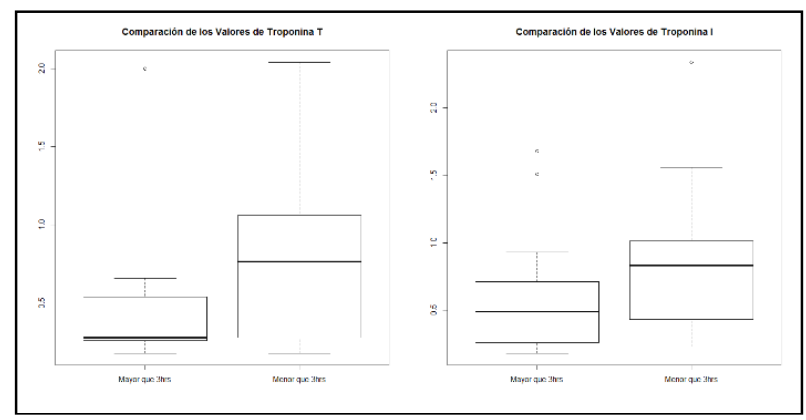

Ilustración 1. Gráfico comparativo de cajas y bigotes de los tiempos de Troponina T e I de acuerdo a los tiempos de elevación. Fuente: Datos del Estudio.

Al relacionar el tiempo de elevación de Troponina con Tipo de Infarto, no se encontró evidencia estadísticamente significativa para asociar directamente la Elevación de la Troponina T e I con la Elevación del ST en el Infarto Agudo del Miocardio $\left(\chi_{T n T}^{2}: 0,43 ; p\right.$-valor: 0,$51 ; \chi_{\text {TnI }}^{2}: 1,15 \mathrm{e}-31 ; \mathrm{p}$-valor: 1 ).

\section{TOPONINA T (TNT) Y TROPONINA I (TNI) Y LOSFACTORES DE RIESGO CARDIOVASCULARESS}

De los pacientes que consumían tabaco $(23 \%)$ el doble presentó Tiempo de Elevación de TnT y Tnl mayor a 3hrs. Es de observar que fue la relación inversa en los pacientes que no consumían tabaco, el porcentaje de pacientes con los tiempos de Elevación de TnT y Tnl menor a 3hrs fue mayor, $54 \%$ y $46 \%$, respectivamente.

Aunque el análisis de frecuencia indica una posible 
relación entre los tiempos de elevación de TnT y Tnl, la prueba de $\chi^{2}$ para TnT vs Tabaquismo no demostró una asociación estadísticamente significativa $\left(\chi_{T n T}^{2}: 1, \chi_{T n T}^{2}\right.$ : 1,62; p-valor: 0,20$)$ igual fue el resultado para $\operatorname{Tnl}\left(\chi_{T n I}^{2}\right.$ : 0,$46 ;$ p-valor: 0,49 )

Tabla 1. Niveles Troponina T e I vs Tipo de Infarto Agudo del M iocardio.

\begin{tabular}{lll}
\hline \multirow{2}{*}{ Troponina T } & \multicolumn{3}{l}{ Infarto Agudo del M iocardio } \\
\cline { 2 - 3 } & Con elevación del ST & Sin elevación del ST \\
\hline Mayor que 3hrs & $6(17,14 \%)$ & $7(20,00 \%)$ \\
Menor que 3hrs & $14(40,00 \%)$ & $8(22,86 \%)$ \\
\hline \multirow{2}{*}{ Troponina I } & \multicolumn{2}{l}{ Infarto Agudo del M iocardio } \\
\cline { 2 - 3 } & Con elevación del ST & Sin elevación del ST \\
\hline Mayor que 3hrs & $9(25,71 \%)$ & $7(20,00 \%)$ \\
Menor que 3hrs & $11(31,43 \%)$ & $8(22,86 \%)$ \\
\hline Fuente: Autres & &
\end{tabular}

Tabla 2. Niveles Troponina T e I vs Tabaquismo.

\begin{tabular}{lll}
\hline \multirow{2}{*}{ Troponina T } & Tabaquismo & \\
\cline { 2 - 3 } & No & Sí \\
\hline M ayor que 3hrs & $8(22,86 \%)$ & $5(14,29 \%)$ \\
Menor que 3hrs & $19(54,29 \%)$ & $3(8,57 \%)$ \\
\hline \multirow{2}{*}{ Troponina I } & Tabaquismo & \\
\cline { 2 - 3 } & No & Sí \\
\hline M ayor que 3hrs & $11(31,43 \%)$ & $5(14,29 \%)$ \\
M enor que 3hrs & $16(45,71 \%)$ & $3(8,57 \%)$ \\
\hline Fuente: Autores & &
\end{tabular}

Tabla 3 Niveles Troponina T e I vs Sedentarismo.

\begin{tabular}{lll}
\hline \multirow{2}{*}{ Troponina T } & \multicolumn{2}{l}{ Sedentarismo } \\
\cline { 2 - 3 } & No & Sí \\
\hline Mayor que 3hrs & $1(2,86 \%)$ & $12(34,29 \%)$ \\
Menor que 3hrs & $6(17,14 \%)$ & $16(45,71 \%)$ \\
\hline Troponina I & Sedentarismo & \\
\cline { 2 - 3 } & No & Sí \\
\hline Mayor que 3hrs & $2(5,71 \%)$ & $14(40,00 \%)$ \\
Menor que 3hrs & $5(14,29 \%)$ & $14(40,00 \%)$ \\
\hline Fuente: Auttores & &
\end{tabular}

El $80 \%$ de los pacientes son sedentarios. Ambos presentaron proporciones similares en los rangos de elevación de TnT y Tnl, tanto en tiempo elevación como en actividad física. Al relacionar los tiempos de elevación para TnT con relación al sedentarismo, no se encontró una asociación positiva $\left(\chi_{T n T}^{2}: 0,93 ; p\right.$-valor: 0,34$)$. Así mismo no se encontró una asociación positiva para la elevación en tiempo de $\mathrm{Tn} I$ con relación al sedentarismo $\left(\chi_{\text {TnI }}^{2}: 0,35 ; p\right.$-valor: 0,54$)$.

Es de observar que si bien no se encontró una asociación estadística entre el tiempo de elevación de TnT y $\mathrm{TnI}$ con respecto al sedentarismo, es claro que si es un factor de riesgo para cualquier tipo de Infarto Agudo al M iocardio con $\left(\chi^{2}: 6,56 ; p\right.$-valor: 0,01$)$.

Tabla 4 Niveles Troponina T e I vs Diabetes.

\begin{tabular}{lll}
\hline \multirow{2}{*}{ Troponina T } & Diabetes & \\
\cline { 2 - 3 } & No & Sí \\
\hline Mayor que 3hrs & $8(22,86 \%)$ & $5(14,29 \%)$ \\
Menor que 3hrs & $13(37,14 \%)$ & $9(25,71 \%)$ \\
\hline \multirow{2}{*}{ Troponina I } & Diabetes & \\
\cline { 2 - 3 } & No & Sí \\
\hline Mayor que 3hrs & $10(28,57 \%)$ & $6(17,14 \%)$ \\
Menor que 3hrs & $11(31,43 \%)$ & $8(22,86 \%)$ \\
\hline Fuente: Autores & &
\end{tabular}

Se encontró que el $40 \%$ de los pacientes tenían Diabetes, para los pacientes diabéticos los tiempos de elevación para TnT y Tnl presentaron una tendencia a ser me que $3 \mathrm{hrs}$ (25,71\% para TnT y 22,86\% para Tnl). Un patrón similar se encontró para los pacientes no diabéticos, donde la elevación tuvo tendencia a darse antes de las 3hrs (37,14\% para TnT y 31,43\% para Tnl).

Los valores de $\chi^{2}$ evidenciaron que el patrón antes era solo una leve tendencia. Indicando contundentemente que ser diabético no tiene una relación con los tiempos de elevación de TnT ( $\chi_{T n T}^{2}$ : 2,53e-31; p-valor: 1$)$ y TnI $\left(\chi_{\text {TnI }}^{2}: 0 ; p\right.$-valor: 1$)$.

Tabla 5 Niveles Troponina T e I vs Hipertensión Arterial.

\begin{tabular}{lll}
\hline \multirow{2}{*}{ Troponina T } & \multicolumn{2}{l}{ Hipertensión Arterial (HTA) } \\
\cline { 2 - 3 } & No & Sí \\
\hline Mayor que 3hrs & $10(28,57 \%)$ & $3(8,57 \%)$ \\
Menor que 3hrs & $7(20,00 \%)$ & $15(42,86 \%)$ \\
\hline \multirow{2}{*}{ Troponina I } & \multicolumn{2}{l}{ Hipertensión Arterial } \\
\cline { 2 - 3 } & No & Sí \\
\hline Mayor que 3hrs & $9(25,71 \%)$ & $7(20,00 \%)$ \\
Menor que 3hrs & $8(22,86 \%)$ & $11(31,43 \%)$ \\
\hline Fuente: Autores & &
\end{tabular}

El 51,43\% de los pacientes con Síndrome Coronario Agudo presentaban HTA diagnosticada. Para el tiempo de elevación de TnT, $42,86 \%$ fue menor a las $3 \mathrm{hrs}$, caso contrario a los pacientes No HTA, quienes en un $28,57 \%$ presentaron un tiempo de elevación de TnT mayor de 3hrs. Similar situación fue para la elevación de la Tnl, para aquellos que fue menor de $3 \mathrm{hrs}$ fueron un $31,43 \%$ de la muestra, es decir aproximadamente un tercio de los pacientes del estudio, para esta misma Troponina - Tnl los pacientes No HTA $(48,57 \%$ ) el $25,71 \%$ presentó una elevación mayora $3 \mathrm{hrs}$.

La prueba de $\chi^{2}$ indicó la relación existente entre la TnT y la HTA $\left(\chi_{T n T}^{2}: 4,97 ; p\right.$-valor: 0,02$)$, relación que fue estadísticamente significativa a un nivel de confianza del 
95\%. En el caso de la Tnl no se encontró asociación estadísticamente significativa $\left(\chi_{\text {TnI }}^{2}: 0,24 ; p\right.$-valor: 0,62$)$.

Tabla 6 Niveles Troponina T e I vs Dislipidemia

\begin{tabular}{lll}
\hline \multirow{2}{*}{ Troponina.T } & \multicolumn{2}{l}{ Dislipidemia } \\
\cline { 2 - 3 } & No & Sí \\
\hline M ayor que 3hrs & $8(22,86 \%)$ & $5(14,29 \%)$ \\
Menor que 3hrs & $13(37,14 \%)$ & $9(25,71 \%)$ \\
\hline Troponina.I & Dislipidemia & \\
\cline { 2 - 3 } & No & Sí \\
\hline M ayor que 3hrs & $10(28,57 \%)$ & $6(17,14 \%)$ \\
Menor que 3hrs & $11(31,43 \%)$ & $8(22,86 \%)$ \\
\hline
\end{tabular}

Es de observar que el $60 \%$ de los pacientes no presentaron dislipidemia elevada. Al contrastar los valores de elevación de $\operatorname{TnT}$ menor que $3 \mathrm{hrs}$, encontramos que la proporción fue similar entre dislipidémicos $(25,71 \%)$ y No dislipidémicos $(37,14 \%)$. Para el tiempo de elevación de $\mathrm{Tn}$ l presentaron un patrón similar a la de la TnT, Siendo mayor para los No dislipidémicos $(31,43 \%)$, sin embargo no siendo grande esta diferencia.

La prueba de $\chi^{2}$ mostró que no hubo una diferencia estadísticamente significativa entre los tiempos de elevación de TnT ( $\chi_{\text {TnT }}^{2}: 2,52 \mathrm{e}-31 ; \mathrm{p}$-valor: 1 ). Así mismo para los tiempo de elevación Tnl ( $\chi_{T n I}^{2}: 0,00 ; p$-valor: 1$)$.

Tabla 7 Niveles Troponina T e I vs Antecedentes de Cardiopatía Isquémica

\begin{tabular}{lll}
\hline \multirow{2}{*}{ Troponina.T } & \multicolumn{3}{l}{ Antecedentes de Cardiopatía Isquémica } \\
\cline { 2 - 3 } & No & Sí \\
\hline M ayor que 3hrs & $11(31,43 \%)$ & $2(5,71 \%)$ \\
Menor que 3hrs & $20(57,14 \%)$ & $2(5,71 \%)$ \\
\hline \multirow{2}{*}{ Troponina.I } & \multicolumn{2}{|l}{ Antecedentes de Cardiopatía Isquémica } \\
\cline { 2 - 3 } & No & Sí \\
\hline M ayor que 3hrs & $13(37,14 \%)$ & $3(8,57 \%)$ \\
Menor que 3hrs & $18(51,43 \%)$ & $1(2,86 \%)$ \\
\hline Fuente: Autores & \multicolumn{2}{l}{}
\end{tabular}

El $88,57 \%$ de los pacientes no tenían antecedentes de cardiopatía isquémica, solo 4 pacientes presentaron este factor de riesgo. Razón por la cual el resultado del $\chi^{2}$ para los tiempos de elevación de ambas tropon inas (TnT y Tnl), no presentaron asociación $\left(\chi_{T n T}^{2}: 2,47 \mathrm{e}-04\right.$; $p$-valor: 0,98; $\chi_{T n I}^{2}: 0,52 ;$-valor: 0,47$)$.

Al realizar la asociación de las principales complicaciones con los tiempos de elevación de TnT y Tnl a través de una prueba multivariada, denominada análisis de correspondencia simples, se encontró una asociación proximal entre el Edema Pulmonar y la Elevación de TnT menor que $3 \mathrm{hrs}$. Y menos proximal pero concluyente entre la Tnl y Taquiarritmias Ventriculares.

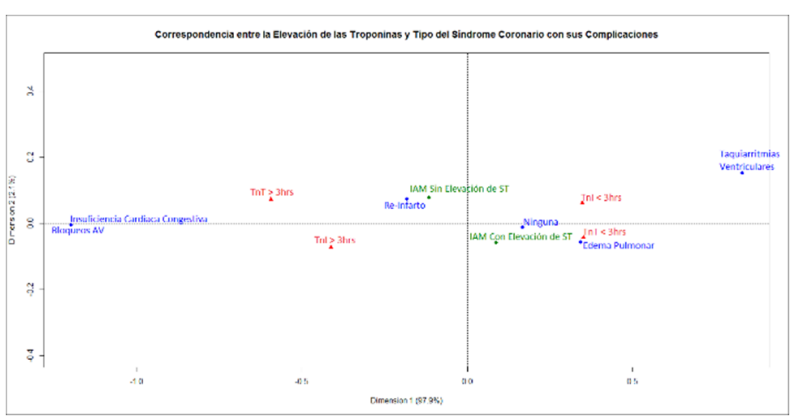

Ilustración 2 Análisis de Correspondencias Simples para la Elevación de Troponina vs Tipo de Síndrome Coronario y las Complicaciones asociadas a este. Fuente: Datos del Estudio.

También es de observar que aunque fue poco proximal se encontraban dentro del mismo plano el tiempo de elevación de TnT mayor que 3hrs con las Insuficiencia Cardiaca Congestiva, Bloqueos AV y Re-infarto.

Tabla 1 Asociación entre los tiempos de elevación de TnT y Tnl con las Complicaciones post-evento coronario.

\begin{tabular}{|c|c|c|c|c|}
\hline \multirow[b]{2}{*}{ Complicación } & \multicolumn{2}{|l|}{ Troponina T } & \multicolumn{2}{|l|}{ Troponina T } \\
\hline & $\begin{array}{c}\text { Mayor que } \\
3 \mathrm{hrs}\end{array}$ & $\begin{array}{c}\text { Menor que } \\
\text { 3hrs }\end{array}$ & $\begin{array}{c}\text { Mayor que } \\
\text { 3hrs }\end{array}$ & $\begin{array}{c}\text { Menor que } \\
\text { 3hrs }\end{array}$ \\
\hline IAM ST elevado & $6(17,14 \%)$ & $14(40,00 \%)$ & $9(25,71 \%)$ & $11(31,43 \%)$ \\
\hline IAM sin ST elevado & $7(20,00 \%)$ & $8(22,86 \%)$ & $7(20,00 \%)$ & $8(22,86 \%)$ \\
\hline Bloqueos AV & $3(8,57 \%)$ & $0(0,00 \%)$ & $3(8,57 \%)$ & $0(0,00 \%)$ \\
\hline Edema Pulmonar & $1(2,86 \%)$ & $5(14,29 \%)$ & $2(5,71 \%)$ & $4(11,43 \%)$ \\
\hline $\begin{array}{l}\text { Insuficiencia Cardiaca } \\
\text { Congestiva }\end{array}$ & $3(8,57 \%)$ & $0(0,00 \%)$ & $3(8,57 \%)$ & $0(0,00 \%)$ \\
\hline Re-infarto & $1(2,86 \%)$ & $1(2,86 \%)$ & $1(2,86 \%)$ & $1(2,86 \%)$ \\
\hline $\begin{array}{l}\text { Taquiarritmias } \\
\text { Ventriculares }\end{array}$ & $0(0,00 \%)$ & $3(8,57 \%)$ & $0(0,00 \%)$ & $3(8,57 \%)$ \\
\hline Ninguna & $5(14,29 \%)$ & $13(37,14 \%)$ & $7(20,00 \%)$ & $11(31,43 \%)$ \\
\hline
\end{tabular}

\section{DISCUSIÓN}

De acuerdo a los datos arrojados en este estudio es claro resalar la sensibilidad de las troponinas en el Síndrome coronario agudo, con porcentajes estadísticos muy similares a la literatura mundial. Los cambios seriados de la TnT y Tnl en un paciente pueden atribuirse a causas patológicas cuando el cambio es superior al valor de referencia calculado en individuos sanos; de manera análoga, un cambio seriado superior al valor de referencia observado en individuos con un trastorno crónico estable indicará un episodio agudo en curso.

Es necesario tener precaución al interpretar estas cifras. En primer lugar, los valores dependen del método 
empleado para determinar la TnT y Tnl, es de notar la atención que el tiempo de elevación de las troponinas tanto la $\mathrm{T}$ y I, no son estadísticamente significativo cuando se compara con el tipo de Síndrome coronario agudo y las complicaciones cardiacas, sin embargo a la hora de analizar los factores de riesgo cardiovascular detectamos como la hipertensión arterial se presentó en el $51,43 \%$ de los pacientes con Síndrome Coronario Agudo.

Mientras que el tiempo de elevación de TnT fue de un $42,86 \%$ antes de las 3hrs en comparación con la Tnl fue de $31,43 \%$ lo que plantea qué relación hay entre la TnT y la hipertensión arterial, esto se puede explicar por La alteración de la función endotelial, que se manifiesta por el desorden del control del tono vasomotor, tanto en grandes arterias y venas como en la microvasculatura, la disfunción endotelial no sólo es un marcador de enfermedad vascular sino que además contribuye a la progresión de la misma.

En la hipertensión arterial el endotelio vascular está deteriorado y promueve cambios funcionales de la pared vascular. Los pacientes hipertensos tienen deprimida la relajación dependiente de endotelio y este trastorno está asociado a una menor bioactividad del óxido nítrico, Si la disfunción endotelial es promotora de hipertensión, esto podría explicar la asociación entre hipertensión y otras patologías que alteran la función endotelial como el Síndrome coronario agudo, en contraste con la literatura mundial en donde el factor de riesgo que incide de manera directa es la dislipidemia y la diabetes en $59.6 \%$ de los casos.

Por otro lado ambas troponinas son sensibles para el diagnóstico del IAM con y sin elevación del ST no hubo significancia estadística, lo que conlleva a pensar que las dos son una herramienta diagnostica importante en esta entidad.

En cuanto al tipo de Síndrome Coronario Agudo y sus complicaciones relacionada con el tiempo de elevación de las TnT menor a 3 horas se evidencio que para el IAMEST la complicación más frecuente fue el edema pulmonar en contraste con el IAMSEST que se relación con la insuficiencia cardiaca congestiva y los bloqueos auriculoventriculares, si se extrapola estos resultados al plano mundial el IAMEST de cara inferior es el que más relación tiene con los bloqueos auriculoventriculares por su extensión al ventrículo derecho y compromiso del nodo.
Por último este tipo de patologías que impactan en la mortalidad de nuestra población colombiana es necesario que el estado cree políticas públicas estatales enfocadas en una mayor inversión, en promoción y prevención en salud.

\section{REFERENCIASBIBLIOGRÁFICA}

1. Hospital TG. A comparison of recombinant hirudin with heparin for the treatment of acute coronary syndromes. The Global Use of Strategies to Open Occluded Coronary Arteries (GUSTO) Ilb investigators. [Internet]. The New England journal of medicine. Wiley Online Library; 1996. Available from: http://onlinelibrary.wiley.com/doi/10.1111/j.13652699.2006.01594.x/full

2. Adams JE, AbendscheinDR, Jaffe AS. Biochemical markers of myocardial injury. Is MB creatine kinase the choice for the 1990s? Circulation. 1993;88(2):750-63.

3. Alexander JH, Sparapani RA, Mahaffey KW, DeckersJW, Newby LK, OhmanEM, et al. Association between minor elevations of creatine kinase-MB level and mortality in patients with acute coronary syndromes without ST-segment elevation. PURSUIT Steering Committee. Platelet Glycoprotein Ilb/IIla in Unstable Angina: Receptor Suppression Usin. JAMA. 2000;283(3):347-53.

4. AntmanEM, Cohen $\mathrm{M}$, BerninkPJ, McCabe $\mathrm{CH}$, Horacek T, Papuchis G, et al. The TIM I risk score for unstable angina/non-ST elevation MI: A method for prognostication and therapeutic decision making. JAMA: the journal of the American Medical Association. 2000.

5. AntmanEM, LouwerenburgHW, BaarsHF, Wesdorp $\mathrm{JCL}$, Hamer B, Bassand JP, et al. Enoxaparin as adjunctive antithrombin therapy for ST-elevation myocardial infarction: Results of the ENTIREthrombolysis in myocardial infarction (TIM I) 23 trial. Circulation. 2002;105(14):1642-9.

6. AntmanEM, McCabe $\mathrm{CH}$, Gurfinkel EP, Turpie AG, BerninkPJ, Salein D, et al. Enoxaparin prevents death and cardiac ischemic events in unstable angina/nonQ-wave myocardial infarction. Results of the thrombolysis in myocardial infarction (TIMI) 11B trial. Circulation. 1999.

7. AntmanEM, Morrow DA, McCabe CH, Murphy SA, Ruda M, Sadowski Z, et al. Enoxaparin versus unfractionated heparin with fibrinolysis for STelevation myocardial infarction. $N$ Engl J Med. 2006;354(14):1477-88. 
8. Apple FS. Acute myocardial infarction and coronary reperfusion. Serum cardiac markers for the 1990s. American Journal of Clinical Pathology. 1992. p. 21726.

9. Hjalmarson A, Herlitz J, Holmberg $S$, Rydén L, Swedberg K, Vedin A, et al. The Göteborgmetoprolol trial. Effects on mortality and morbidity in acute myocardial infarction. Circulation. 1983.

10. Bhatt DL, Hirsch AT, Ringleb PA, Hacke W, TopolEJ. Reduction in the need for hospitalization for recurrent ischemic events and bleeding with clopidogrel instead of aspirin. Am Heart J. 2000;140(1):67-73.

11. Boden WE, O'Rourke RA, Crawford MH, Blaustein AS, Deedwania PC, ZobleRG, et al. Outcomes in patients with acute non-Q-wave myocardial infarction randomly assigned to an invasive as compared with a conservative management strategy. Veterans Affairs Non-Q-Wave Infarction Strategies in Hospital (VANQWISH) Trial Investigators. The New England journal of medicine. 1998.

12. Braunwald E, Jones RH, Mark DB, Brown J, Brown L, Cheitlin MD, et al. Diagnosing and managing unstable angina. Agency for Health Care Policy and Research. Circulation. 1994;90(1):613-22.

13. Braunwald E, AntmanEM, Beasley JW, CaliffRM, Cheitlin MD, HochmanJS, et al. ACC/AHA guidelines for the management of patients with unstable angina and non-ST-segment elevation myocardial infarction: executive summary and recommendations. A report of the American College of Cardiology/American Heart Association task force on pract. Circulation. 2000;102(10):1193-209.

14. Brouwer MA, M artin JS, M aynard C, Wirkus M, Litwin $P E$, Verheugt $F W$, et al. Influence of early prehospital thrombolysis on mortality and event-free survival (the Myocardial Infarction Triage and Intervention [M ITI] Randomized Trial). M ITI Project Investigators. The American journal of cardiology. 1996.

15. Cannon CP, WeintraubWS, Demopoulos LA, Robertson DH, GormleyG], Braunwald E. Invasive versus conservative strategies in unstable angina and non-Q-wave myocardial infarction following treatment with tirofiban: rationale and study design of the international TACTICS-TIMI 18 Trial. Treat Angina with Aggrastat and determine Cost of The American journal of cardiology. 1998.

16. Mann DL, Zipes DP, Libby P, Bonow RO. Braunwald's Heart Disease: A Textbook of Cardiovascular Medicine. 10th ed. Elsevier; 2015. 2128 p.

17. Chaitman BR, Stone PH, Knatterud GL, Forman SA,
Sopko G, Bourassa M G, et al. Asymptomatic cardiac ischemia pilot (ACIP) study: Impact of anti-ischemia therapy on 12-week rest electrocardiogram and exercise test outcomes. J Am CollCardiol. 1995;26(3):585-93.

18. Christenson RH, Azzazy HM . Biochemical markers of the acute coronary syndromes. Clin Chem. 1998;44(8 Pt 2):1855-64.

19. Cohen M, Demers C, Gurfinkel EP, Turpie AG, FromellGJ, Goodman S, et al. A comparison of lowmolecular-weight heparin with unfractionated heparin for unstable coronary artery disease. Efficacy and Safety of Subcutaneous Enoxaparin in Non-QWave Coronary Events Study Group. The New England journal of medicine. 1997.

20. Cohen $M$, Théroux P, Borzak S, Frey MJ, White HD, Van Mieghem W, et al. Randomized double-blind safety study of enoxaparin versus unfractionated heparin in patients with non-ST-segment elevation acute coronary syndromes treated with tirofiban and aspirin: The ACUTE II study. Am Heart J. 2002;144(3):470-7.

21. Chen Z, Xie J. Early intravenous then oral metoprolol in 45852 patients with acute myocardial infarction: Randomised placebo-controlled trial. Lancet. 2005;366(9497):1622-32.

22. Hansen JF. Effect of verapamil on mortality and major events after acute myocardial infarction (The Danish Verapamil Infarction Trial II - DAVIT II). Am J Cardiol. 1990;66(10):779-85.

23. ScanlonPJ, FaxonDP, Audet A-M, Carabello B, DehmerG], Eagle KA, et al. ACC/AHA Guidelines for Coronary Angiography: Executive Summary and Recommendations: A Report of the American College of Cardiology/American Heart Association Task Force on Practice Guidelines (Committee on Coronary Angiography) Developed in collaboration. Circulation. 1999. p. 2345-57. 